\title{
How to Predict Earthquakes with Microsequences and Reversed Phase Repetitive Patterns
}

\author{
Giulio Riga1, Paolo Balocchi² \\ ${ }^{1}$ Geologist, Independent Researcher, Lamezia Terme, Italy \\ ${ }^{2}$ Geologist, Independent Researcher, Modena, Italy \\ Email: giulio.riga@tin.it
}

Received 31 May 2016; accepted 6 July 2016; published 9 July 2016

Copyright (C) 2016 by authors and Scientific Research Publishing Inc.

This work is licensed under the Creative Commons Attribution International License (CC BY).

http://creativecommons.org/licenses/by/4.0/

(c) (i) Open Access

\begin{abstract}
A strong earthquake is always preceded by groupings of shocks whose identification and understanding constitute a sound method for improving short-term earthquake forecasts. Thanks to a graphical method, we have identified and classified some microsequences and reversed phase repetitive patterns that precede the hazardous events. The seismic microsequences include a series of information useful to know in advance the beginning of energy release and accumulation phases that usually precede and follow a moderate-to-high magnitude earthquake. Their identification and correct interpretation allow us to determine various warning signals. In particular, through the analysis of their shape and position in the seismic sequence we can claim that the strongest earthquakes occur shortly after the formation of some peculiar micro-sequences. The checks carried out on large data sets related to earthquakes occurred in the past have shown that the analysis procedures developed do not depend on the size of the area analyzed while predicting a high percentage of moderate-to-high magnitude earthquakes.
\end{abstract}

\section{Keywords}

Microsequences, Foreshocks, Pattern, Triggers, Earthquake, Mainshock

\section{Introduction}

In any earthquake prediction study, it is essential to identify the precursory phenomena within the seismic data to be analyzed [1]. To this end, it is necessary to analyze a solid scientific database to identify and classify the premonitory models that precede big seismic events, whose nature and shape, variable from one event to another, 
are probably controlled by the tectonic environment (geometries of the fault, strain rate) and the fault plan heterogeneity [2]. The seismic sequence of a given area includes all the information needed to understand its evolution in space and time [3]; through an in-depth analysis of its inner structure it is possible to draw conclusions related to strong events prediction. In fact, the long-term sequence includes smaller-scale, medium-term sequences, which, in turn, contain short- and imminent-term sequences that influence the sequence evolution in relation to development speed and magnitude (space-time evolution).

The analysis performed on several seismic sequences has identified in short and imminent-term time windows, particular groupings of shocks (microsequences and repetitive patterns), which anticipate a phase inversion [4]. Usually, micro-sequences and repetitive patterns are formed before the beginning/end of the energy release energy, since the distribution of shocks over time is not random but follows rules that allow us to understand the development level achieved by the sequence and to make more reliable predictions.

The analysis of the seismic sequence structure is performed using: a) a spatial coverage of variable datasets ranging from regional scale to international scale (or global, which covers an area ranging from few tens of $\mathrm{km}^{2}$ to thousands of $\mathrm{km}^{2}$ ); b) a range of magnitude values of 2.0 - $10 \mathrm{M}$; (c) a range of depths of 1 - $50 \mathrm{~km}$.

\section{Methodology}

\subsection{Microsequences TT-7S and DB-3SE}

We all are aware of the relative importance of the small and large earthquakes to change static stresses and trigger earthquakes. The small shocks observed before strong earthquakes, suggest that they have specific properties that can be used as of earthquakes precursors. Even though big earthquakes are unquestionably more important compared to smaller ones in relation to the energy release, small shocks collectively have the same influence [5].

Microsequences TT-7S (Triple Maximum-Seven Shocks) and DB-3SE (Double Minimum-Three small shocks) are graphic patterns that develop inside the seismic sequence and allow us to locate the point of closure/beginning of an energy release phase.

In microsequence TT-7S (Figure 1), we identify a peculiar trend, which involves the magnitude values fluctuations that is completed during the energy release phase: it develops a first maximum (point 2) followed by a minimum value (point 3). Hereinafter, the magnitude values begin to rise again (point 4) reaching higher values than the previous maximum, and a second minimum (point 5), which generally has greater value compared to the previous one. The next step is characterized by a third peak (point 6) lower than the second maximum, followed by the last minimum (point 7) that is placed below the "transition line” [6] joining the minimum points 3 and 5, respectively. This completes the microsequence TT-7S [7].

Overall, the microsequenceTT-7S appears as a strong fluctuation of the magnitude values that occur and are completed during the energy release phase.

In Figure 2 the green circles indicate the fourth shock in the microsequence whose magnitude $M(4)$ must comply with the following condition:

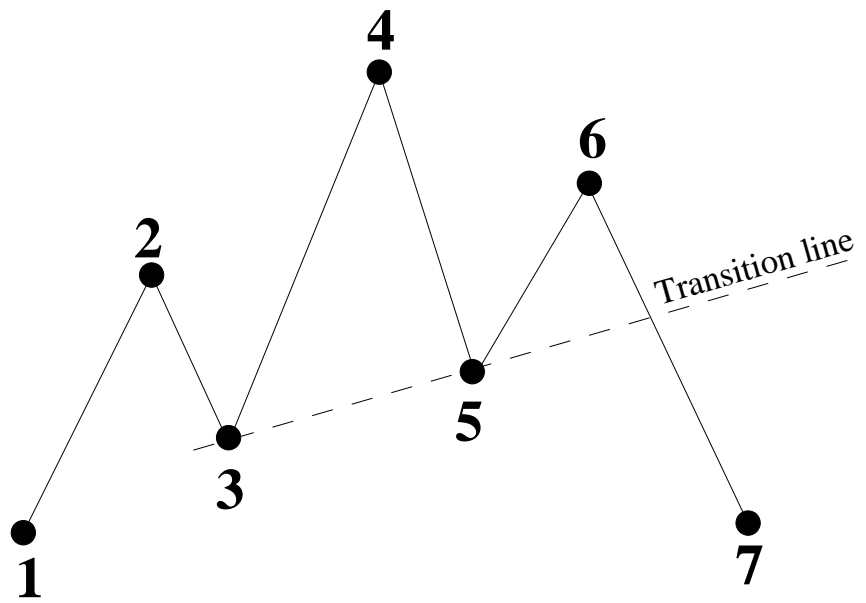

Figure 1. Microsequence TT-7S pattern. 


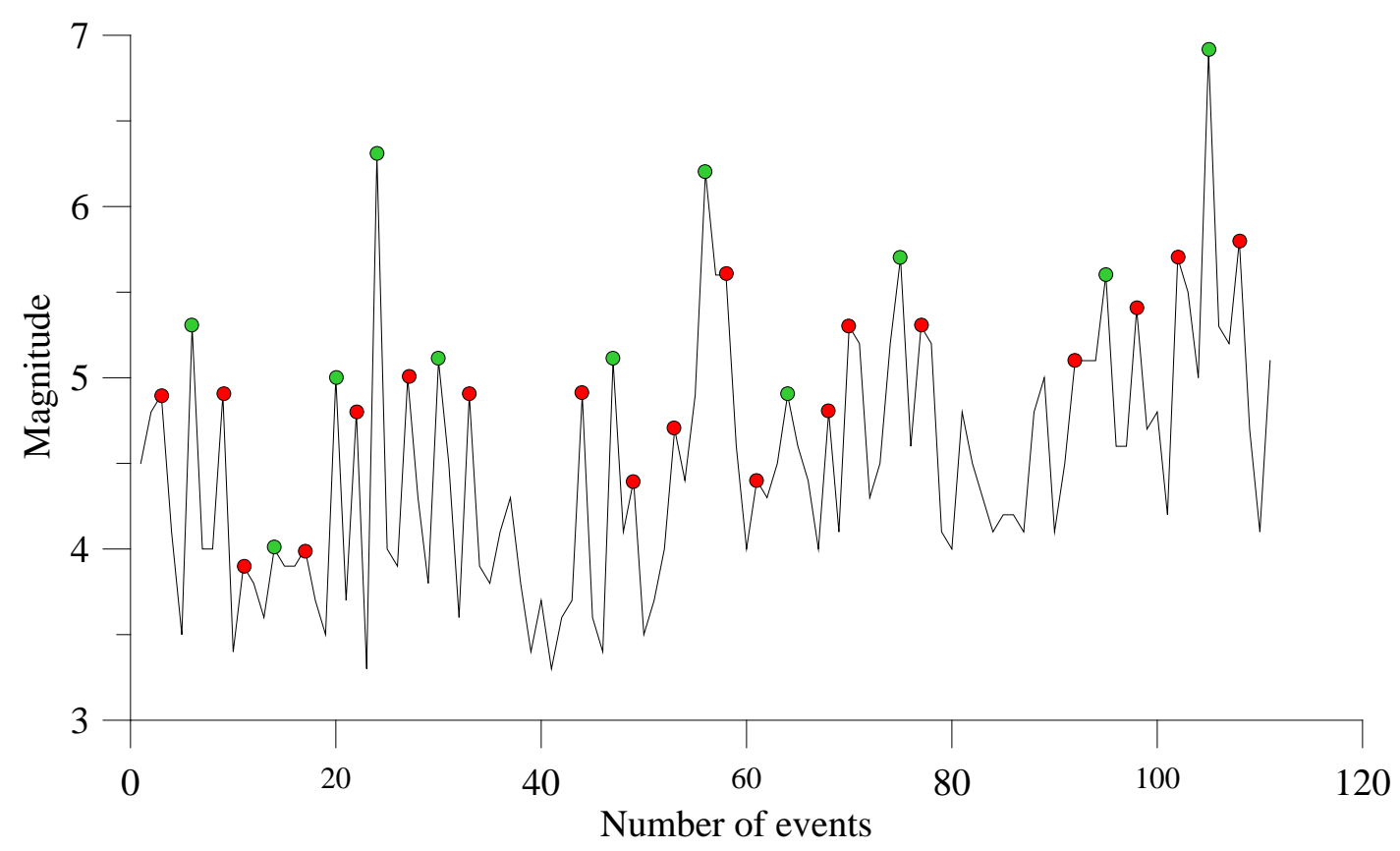

Figure 2. Microsequences TT-7S fluctuations.

$$
M(2) \leq M(4) \geq M(6)
$$

While the red circles indicate the points 2 and 6, respectively.

The microsequence BD-3SE [4] show more reliable features for imminent-term predictions. It is characterized by three small shocks fluctuations within two shocks of greater magnitude (Figure 3), where their completion establishes both the reversal of the energy accumulation phase and a critical condition, in which the successive small shocks trigger either average magnitude or catastrophic events. The microsequence's characteristic lies in the presence of a double minimum (points 1 and 3), and can be either symmetric, if the two minimums have the same magnitude value (Figure 3(a)) or asymmetrical, if the two minimums values are different (Figures 3(b)-(e)).

The microsequence BD-3SE is identifiable on charts concerning both the daily/monthly magnitude values trends or the progressive number of shocks, and generally they are completed after a very short period. Conversely, the release phase is active for one to eight days/months or eight events (in relation to the sequence's development time) after the trigger point, which is represented by the second minimum of the microsequence DB-3SE (red triangle). After the trigger point, it is possible that the magnitude values progressively increase up to the final target, thus generating, a progressive earthquake- or flash-earthquake type energy release phase [4].

The procedure for identifying the microsequence DB-3SE is the following:

1) Identify the absolute maximum and relative values $P(n)$, which represent the extreme points (Figure 4);

2) Identify the extreme points $P_{1}(n)$ (second shock in the microsequence DB-3SE) whose magnitude $M_{1}(n)$ must comply with the following conditions:

$$
\begin{aligned}
& M_{1}(n-1) \geq M_{1}(n)<M_{1}(n+1) \\
& M_{1}(n-1)>M_{1}(n) \leq M_{1}(n+1)
\end{aligned}
$$

where $M_{1}(n-1), M_{1}(n+1)$ are the magnitude values of the extreme point that precedes and follows the point $P_{1}(n)$, respectively (Figure 5).

3) Draw the line joining the second shock in the microsequence DB-3SE (blue line) (Figure 6);

4) Draw the line joining the fourth shock in the microsequence TT-7S (black line).

During a seismic sequence development it is possible to identify the beginning of an energy release phase by assuming the following condition: 


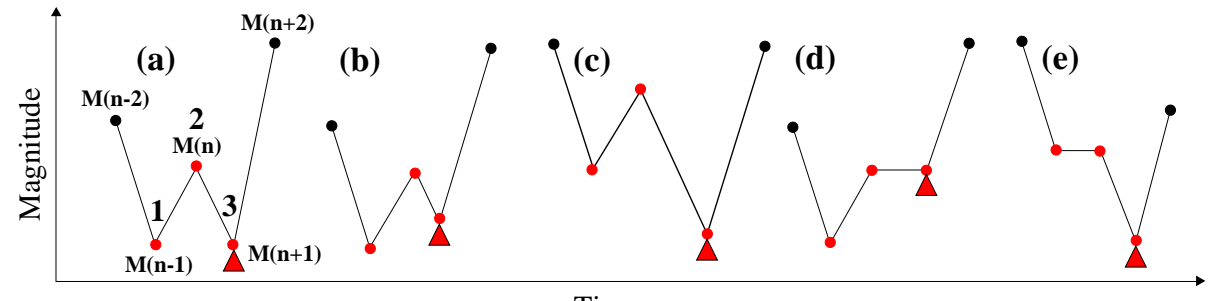

Figure 3. Schematic representation of microsequence DB-3SE. The red circles indicate the microsequence, while the red triangle indicates the trigger point of the energy release phase. The black circles represent the microsequence's starting and completion shocks.

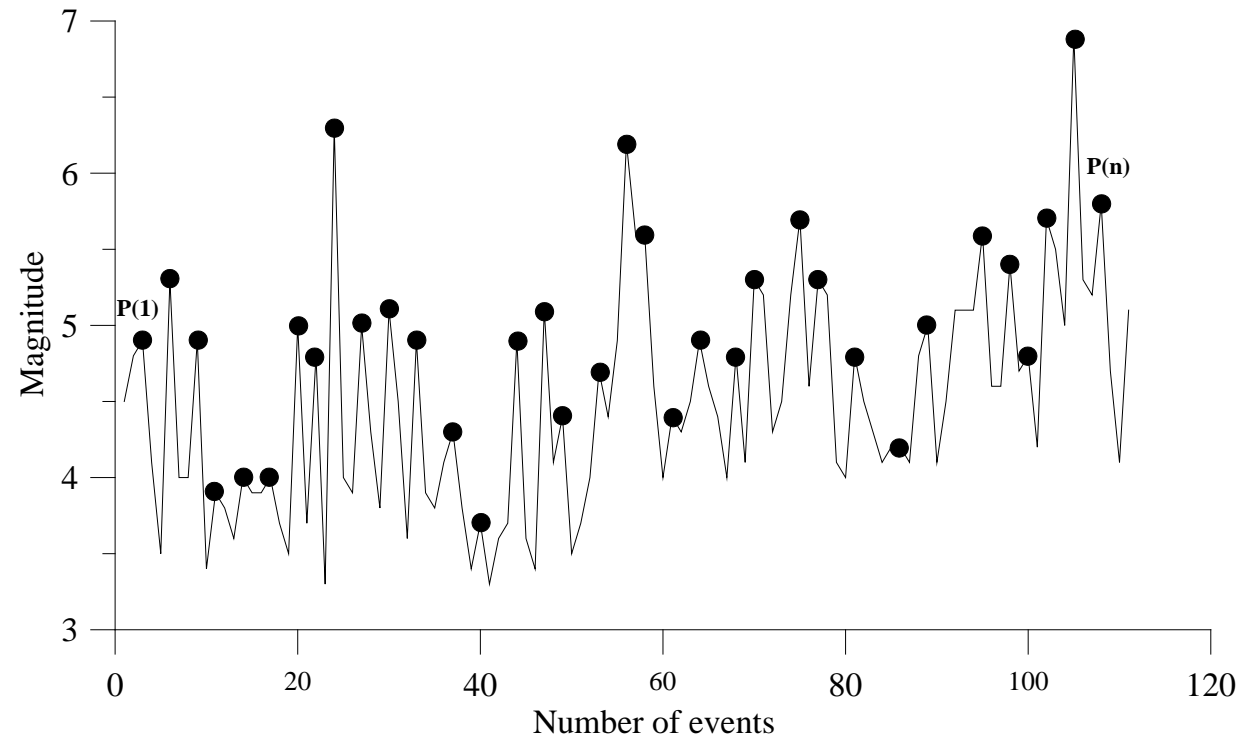

Figure 4. Identification of absolute and relative maximum values (black circles).

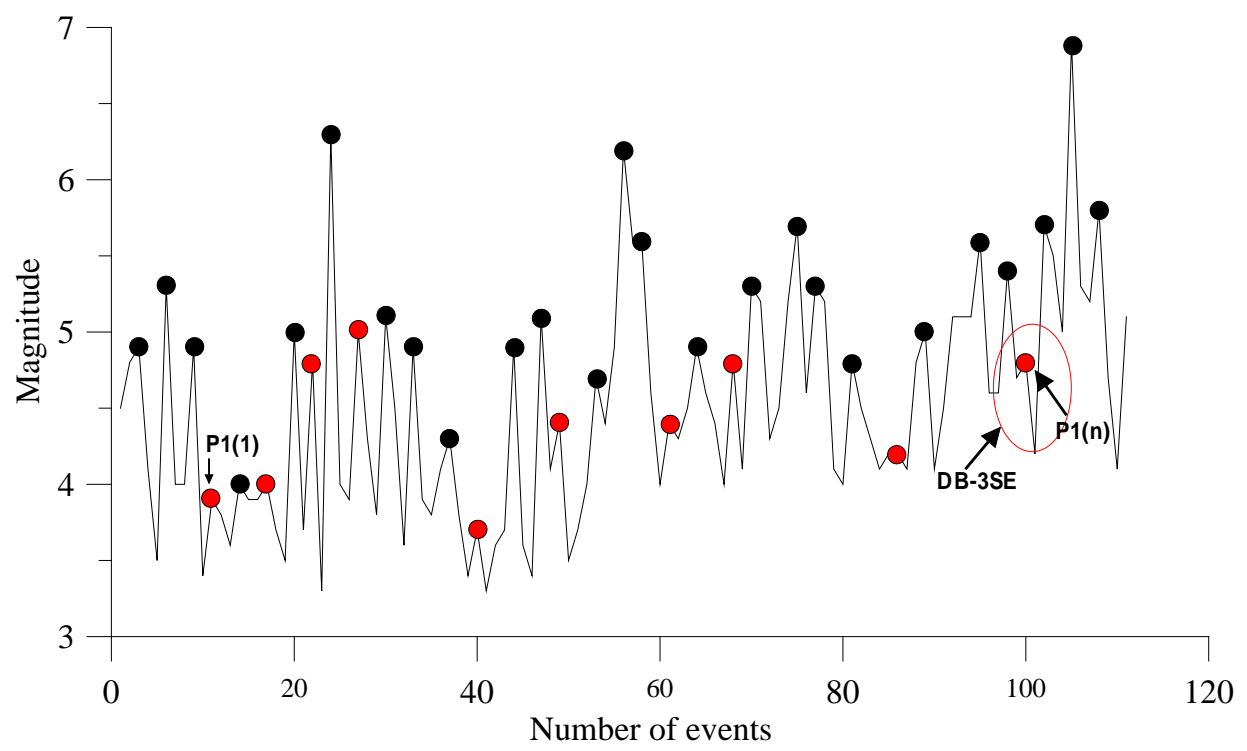

Figure 5. Procedure apt to identify the microsequences DB-3SE. In the red ellipse an example of microsequence DB-3SE is shown. The red circle indicates the second shock in the microsequence. The black circles indicate the extreme points (absolute and relative maximum values)_Earthquake occurred in China on 13 April 2010. 


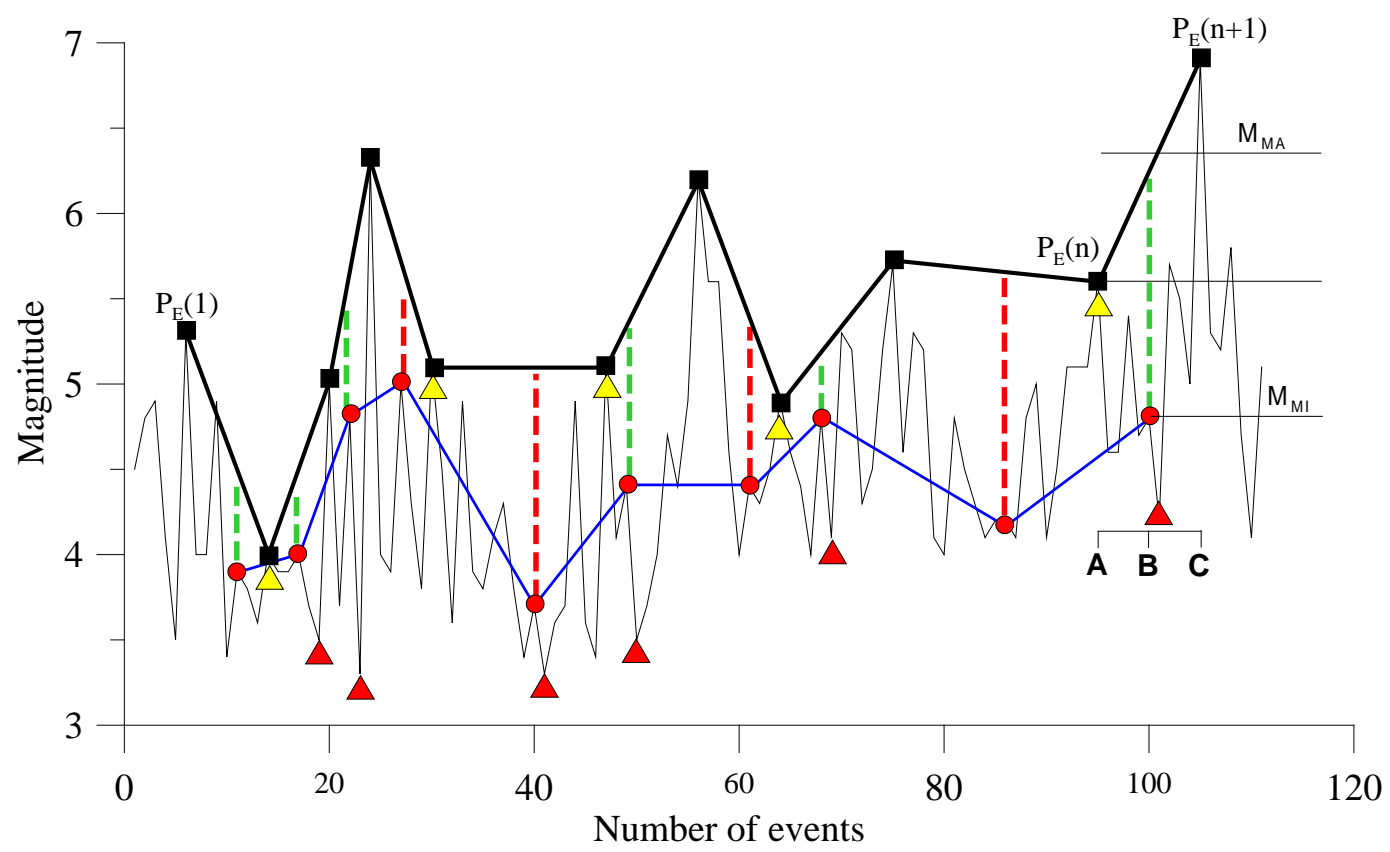

Figure 6. Definition of energy accumulation and release phases. The yellow triangles represent the first-order warning signals, while the red triangles represent the second and third order ones; graphical identification of minimum $\left(M_{M I}\right)$ and maximum magnitude $\left(M_{M A}\right)$.

If $M_{E}(n) \geq M_{E}(n-1)$ is the beginning of an energy release phase (yellow triangles). Where $M_{E}(n)$ is the magnitude of the fourth shock $P_{E}(n)$ in the microsequence TT-7S that must be confirmed by the following condition as well:

$$
A B<B C
$$

where $A B$ is the temporal distance or number of shocks between the point $P_{E}(n)$ (yellow triangle) and the second shock in the microsequence DB-3SE (red circle), while the segment $B C$ represents the temporal distance or the number of shocks between the microsequence DB-3SE and the last recorded event during the current phase.

The $A B>B C$ condition indicates an ongoing energy accumulation phase, while the $A B=B C$ condition is regarded as the "watershed" between the energy accumulation and release phases.

In general, the magnitude value of the second shock in the micorsequence DB-3SE corresponds to the minimum expected magnitude $\left(M_{M I}\right)$ during the energy release phase, while an indicative value of the maximum expected magnitude $\left(M_{M A}\right)$ shall be calculated by adding the difference between the magnitude of the latter point and the minimum expected magnitude value $\left(M_{M I}\right)$ to the magnitude value of the fourth shock in the microsequence TT-7S (point $P_{E}(n)$ ).

In order to obtain a more accurate method, however, in some cases it is necessary to use other types of signals to confirm the ongoing phase. For example, a clear signal of phase inversion is given by the fluctuations of the red line (Figure 7) that joins the extreme points of the seismic sequence to the second shock in the microsequence DB-3SE. In the lower part of the graph, the amplitudes of the imminent-short term energy accumulation and release phases are indicated by red and green horizontal bars.

From the graph it is possible to infer the following information:

1) The microsequence DB-3SE indicates the end of the energy accumulation phase and the beginning of the energy release phase (point $A$ ).

2) Point $B$ represents an imminent-short term foreshock and the first maximum in microsequence TT-7S.

3) The point $C$ represents the mainshock or a stronger shock and the second maximum in microsequence TT-7S.

4) The point D represents the first shock of the energy accumulation (aftershock) and also the third maximum in microsequence TT-7S.

The seismic sequence development takes place according to an evolution that follows this pattern: after the 


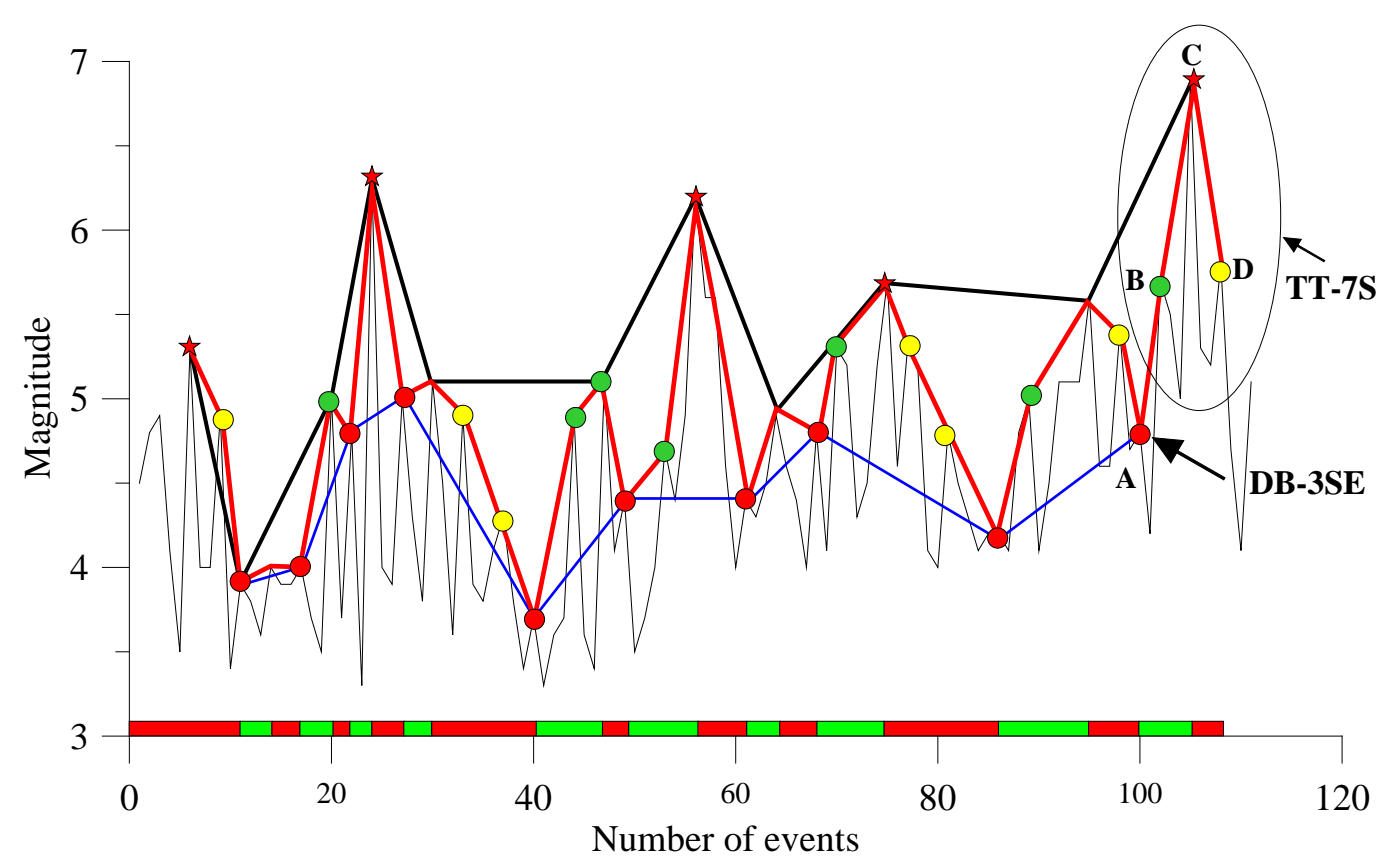

Figure 7. Alternating accumulation and release of energy phases.

microsequence DB-3SE formation (point A), a single shock of greater magnitude than the second shock in microsequence DB-3SE or more shocks whose magnitude increases over time (points B and C) may occur. In point $C$ the closure of the energy release $p$ occurs if the third maximum in the microstructure TT-7S is formed (point D), which may coincide with a second mainshock or with an imminent-term aftershock (yellow circle), while the imminent-term energy accumulation phase ends with the formation of a microsequence DB-3SE.

\subsection{Warning Signs}

A traditional use of the microsequence DB-3SE involves the triggering of a first alert in correspondence of the occurrence of the third shock it consists of. However, this method does not allow to know the extension of the energy release phase.

A more reliable method is to associate alerts in increasing order with the microsequence DB-3SE and with the shocks that follow (Figure 8). For example, the third shock in microsequence DB-3SE that precedes an extreme point $P_{E}(n)$ of magnitude $M_{E}(n) \leq M_{E}(n-1)$ is associated with a first-order alert (green triangle), while the extreme point $P_{E}(n)$ is associated with second order alert (yellow color).

The imminent-term, higher order signals (red triangles) are associated with microsequences DB-3SE that forms after the second order signal.

The graph clearly shows how the warning signs occur following a repetitive 1-2-3 or 1-2-3-4 order that allows to follow the sequence development during the energy release phase and to establish the levels of danger that grow over time.

It is possible to use a different system to define the various order warning signs, using the time/number distance of shocks between the extreme point $P_{E}(n)$ of magnitude $M_{E}(n) \leq M_{E}(n-1)$ and the second shock in microsequence DB-3SE. The extreme point $P_{E}(n)$ constitutes a first order warning sign, the third shock in microsequence DB-3SE is a second-order warning sign, while the point $\mathrm{C}$, placed at a distance from the point $\mathrm{B}$ equal to $A B$, represents a third order alert (Figure 9).

\subsection{Repetitive Patterns}

A strong earthquake is preceded by shocks of different magnitude organized in repetitive patterns that anticipate the energy release phase, whose identification and understanding constitute a sound method to make short and medium term predictions. 


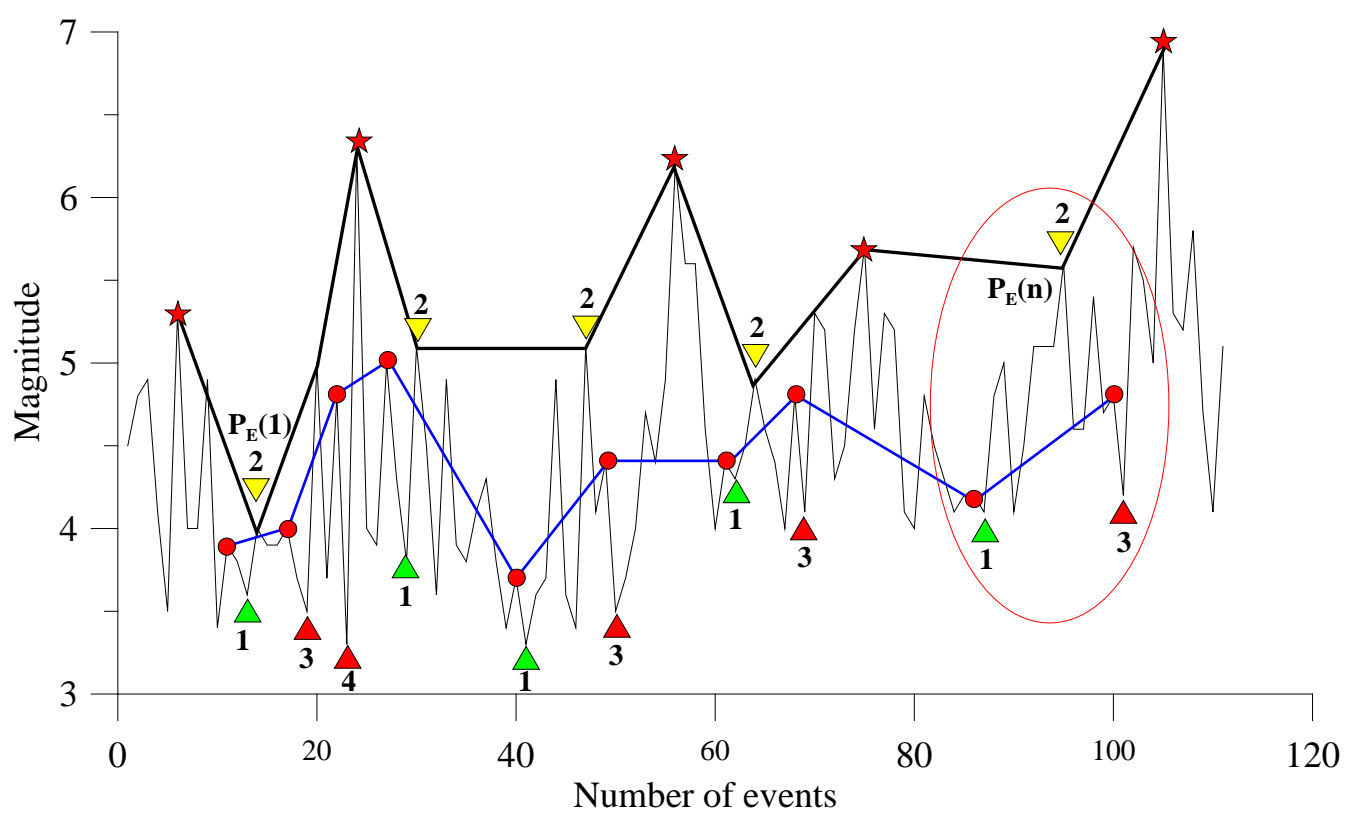

Figure 8. Various orders warning signals. The red star indicate the strongest earthquakes.

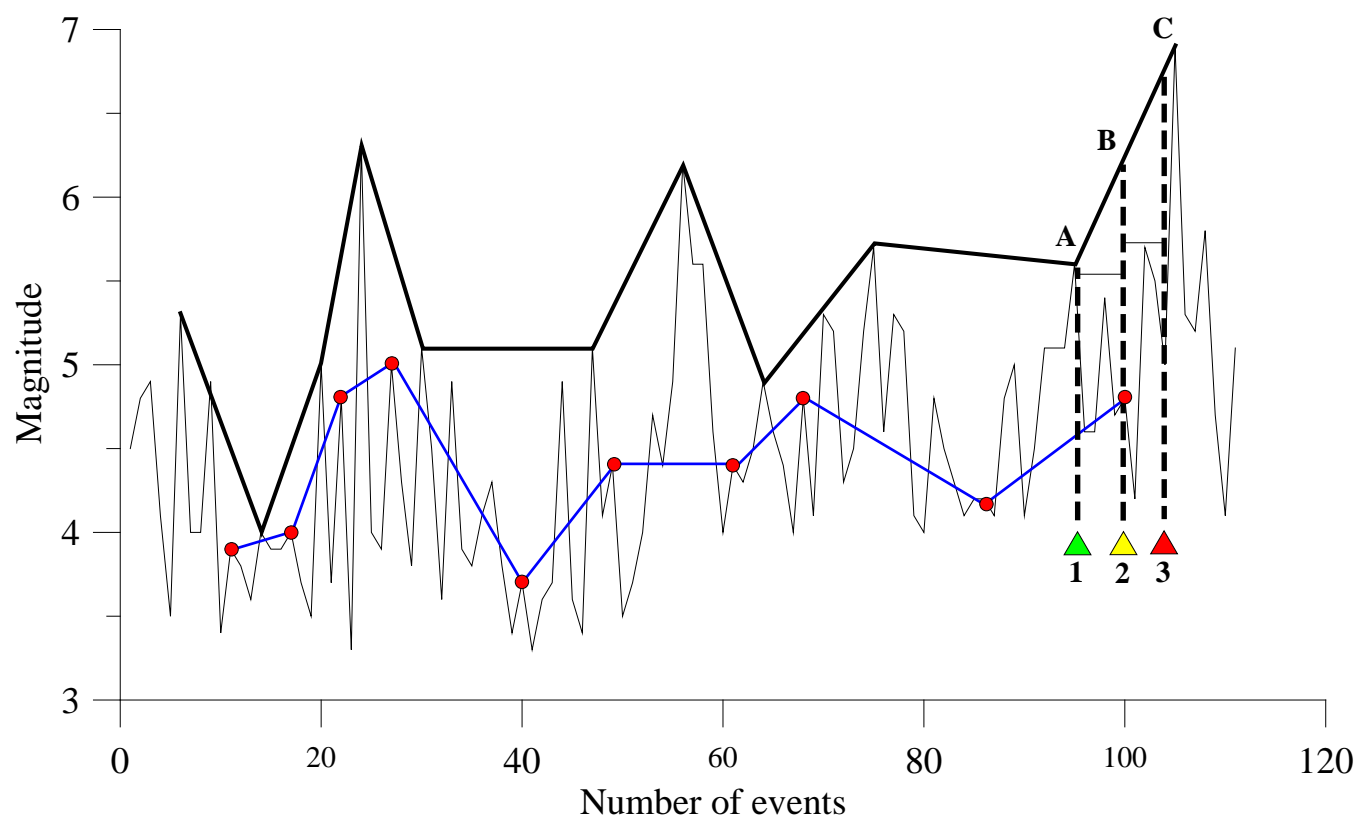

Figure 9. Various order warning signs.

The method for identifying the repetitive patterns consists in drawing the line that joins the fourth shock in microsequence TT-7S (magenta-colored upper line) and the line joining the low extreme points (magenta-colored bottom line) whose magnitude values must comply with the following conditions (Figure 10):

$$
\begin{aligned}
& M_{I}(n-1) \geq M_{I}(n)<M_{I}(n+1) \\
& M_{I}(n-1)>M_{I}(n) \leq M_{I}(n+1)
\end{aligned}
$$

where $M_{I}(n-1)$ and $M_{I}(n+1)$ are the magnitude values of the low extreme points that precede and follow the point $P_{I}(n)$ of magnitudo $M_{I}(n)$.

The two lines constitute the fluctuation band of the extreme points values. 


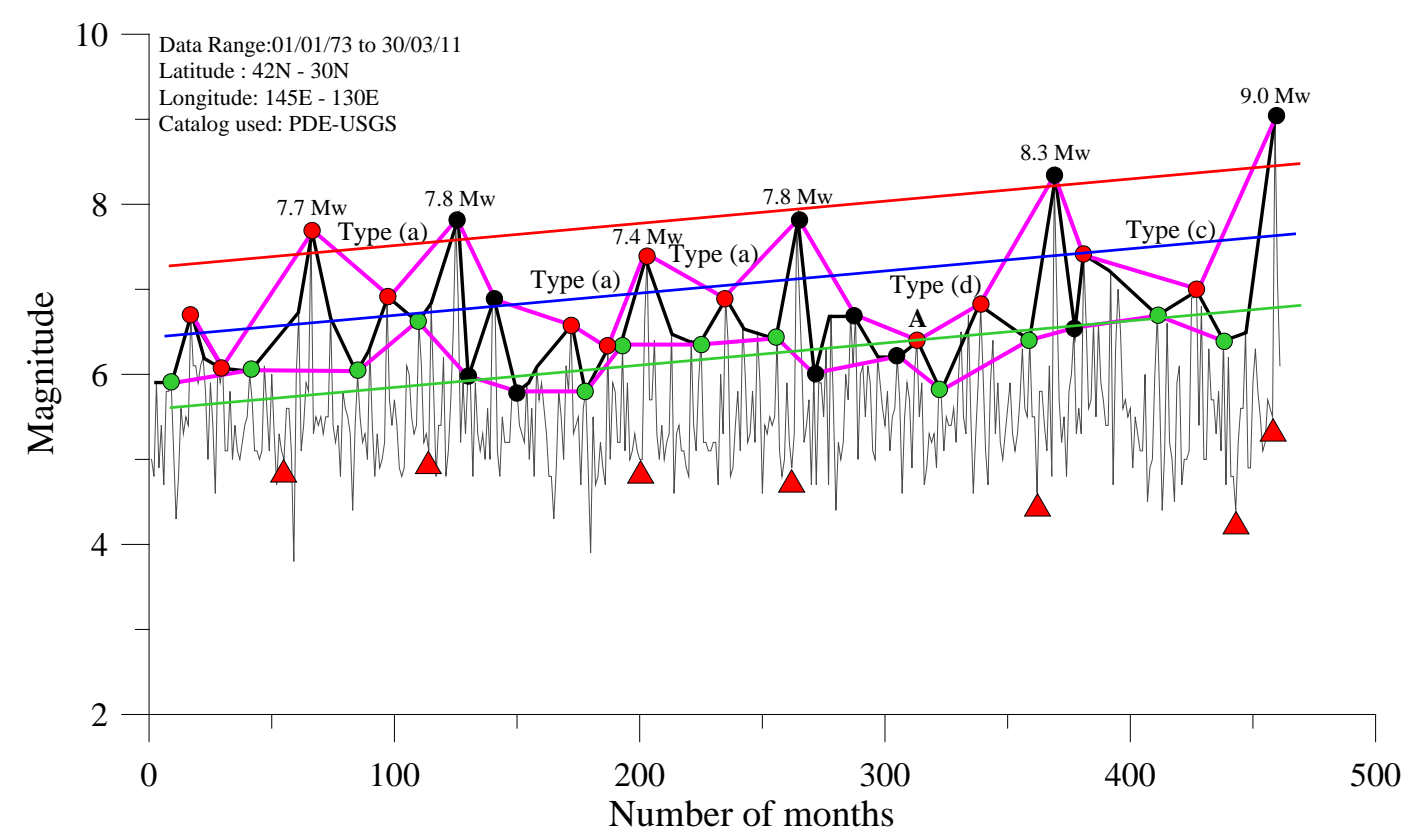

Figure 10. Procedure to identify the repetitive patterns. We observe some structures of repetitive patterns based on the type shown in Figure 11. The red triangles indicate the third-order warning signs generated by the microsequence DB-3SE.

The distinctive feature of the band is that the spaces between the top and the bottom line will vary as a function of the ongoing phase. Basically, the fluctuation band provides information on the ongoing phase in the seismic sequence: if the sequence is currently in an energy release phase then the band undergoes a progressive widening, while if it is in an accumulation phase, the band undergoes a shrinkage. In particular, during the activation step of the energy release phase the band shrinks further to contain the small shock.

The flattening of the fluctuation band usually means that a trigger point of the energy release phase is about to form.

If the magnitude values begin to exceed the interpolation line of the extreme points values (solid blue line), it means that the motion will continue usually upwards (with increasing magnitude values), while a downwards crossing will indicate the presence of an energy accumulation phase.

By default, after a strong earthquake the magnitude values always tend to return below the straight interpolation line.

The band upper line and the straight interpolation line movements provide some information on the magnitude values of the strongest earthquakes.

The method adopted involves the drawing of the parallel line to the interpolation straight line (green line) from the minimum point of the oscillation band upper line (point A) and the projection of a second parallel (line red) on it, whose amplitude is equal to that between the interpolation straight line and the lower parallel line. The magnitude values supplied by the second parallel line over time may be assumed as the strongest earthquakes' expected target.

Figure 11 shows the five repetitive patterns consisting of four shocks (two maximum and two minimum) generated by the oscillation band of the extreme points.

Models $a-b-c$ will form at the end of a energy accumulation cycle, while models $d$-e during the energy release cycle (they are the most important, because the shock S3 is a foreshock).

In addition, the pattern are followed by warning signals generated by the microsequence DB-3SE that anticipate the strongest shocks.

\section{Results}

In Figure 12 shows the results obtained from the application of the methodology explained above to the seismic sequence of the earthquake occurred in 1989 in Loma Prieta. 


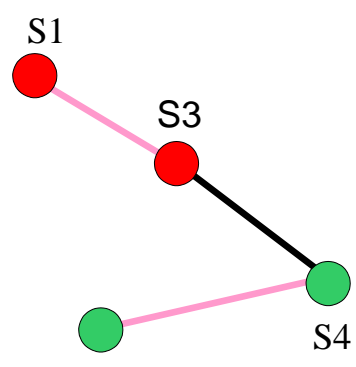

S2

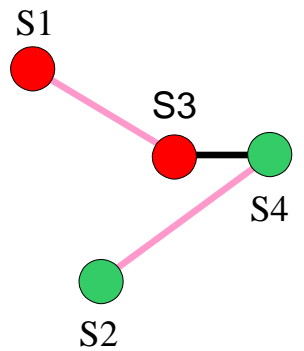

(b)

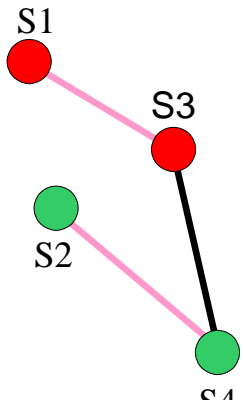

(c)

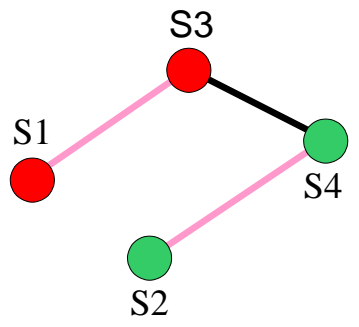

(d)

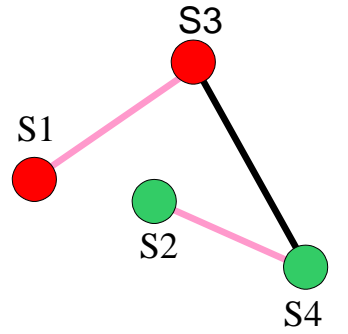

(e)

Figure 11. The repetitive phase inversion patterns.

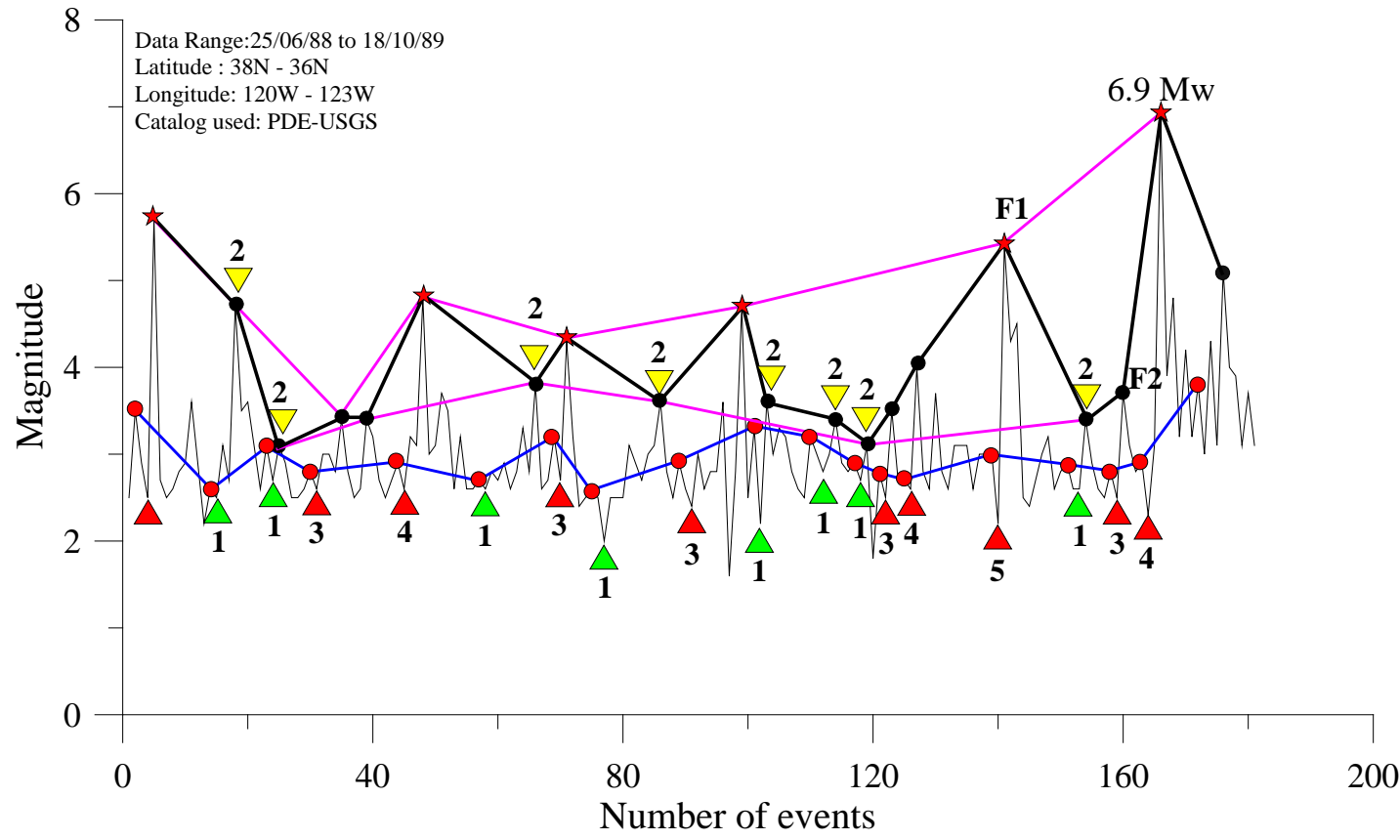

Figure 12. Warning signs before the earthquake in Loma Prieta, 1989.

The main shock occurred on 18 October 1989 (6.9 Mw) was preceded by a progressive earthquake-type energy release phase [6] characterized by a short-term foreshock of magnitude 5.4 ML recorded on 08 August 1989 (point F1) and preceded by a fifth-order imminent-term warning sign generated by the microsequence DB-3SE.

The subsequent evolution of the seismic recordings over time shows the development of an impending-term accumulation phase consisting of shocks of magnitude less than $3.5 \mathrm{M}$, some of which are organized in microsequences DB-3SE that have generated various-order warning signs. 
In particular, we note the formation of a first-order signal (point 1) generated by the third shock in microsequence DB-3SE, which has formed after the first foreshock (point F1), followed by the second-order signal generated by the extreme point (point 2) and by subsequent points (points 3 and 4). The latter preceded respectively the second foreshock (point F2) and the mainshock with magnitude of $6.9 \mathrm{Mw}$, generated by the third shock in microsequence DB-3SE. In addition, prior to the energy release phase, the oscillation band shrinks, while after the first foreshock it expands.

Even in this case, the oscillation band formed by the extreme points lines (black line) and by the second shocks in microsequence DB-3SE (blue line) can be used to identify phase inversion patterns (Figure 13). In fact, this oscillation band is an excellent oscillator to understand when the seismic sequence reaches a critical stage where small shocks can trigger shocks of greater magnitude. Essentially, when the band narrows, we reach the terminal step of the energy accumulation phase: this may suggests that in the short term the magnitude values could begin to grow. The band provides also significant warning signs (you should always bear in mind that this is a predictive analysis, i.e. it tries to predict future events and therefore not yet occurred) generated by the intersection between the descending sections extensions of the line joining the extreme points and the ascending sections extensions of the line joining the second shock in the microsequence DB-3SE (red triangle).

The magnitude minimum value $\left(M_{M I}\right)$ expected in the energy release phase and associated with the warning sign, corresponds to the magnitude value of the extreme point that precedes the signal (green circle).

In order to further reduce the errors related to the interpretation of the signals which inevitably the procedure can generate, we recommend the adoption of one or more filters as the simplified Aroon oscillator [4], with the purpose of identifying the energy accumulation areas in relation to the data provided by the oscillation band.

Starting from the previous example, in the graph shown in Figure 14 various order warning signs are reported (green, yellow and red triangles), which were identified through the distance between the extreme point (black circle) and the second shock in microsequence DB-3SE (red circle).

The first order warning sign coincides with the extreme point. The main shock has been triggered by an asymmetric microsequence DB-3SE (second order signal), while the third-order signal, placed at a distance BC = $\mathrm{AB}$ (point $\mathrm{C}$ ) has formed a few seconds before the mainshock.

The graph displayed in Figure 15 shows some details of the microsequences TT-7S that have formed during the energy release phase and after the most hazardous seismic events.

In particular, it is possible to observe two imminent-term microsequences TT-7S in which the second maximum

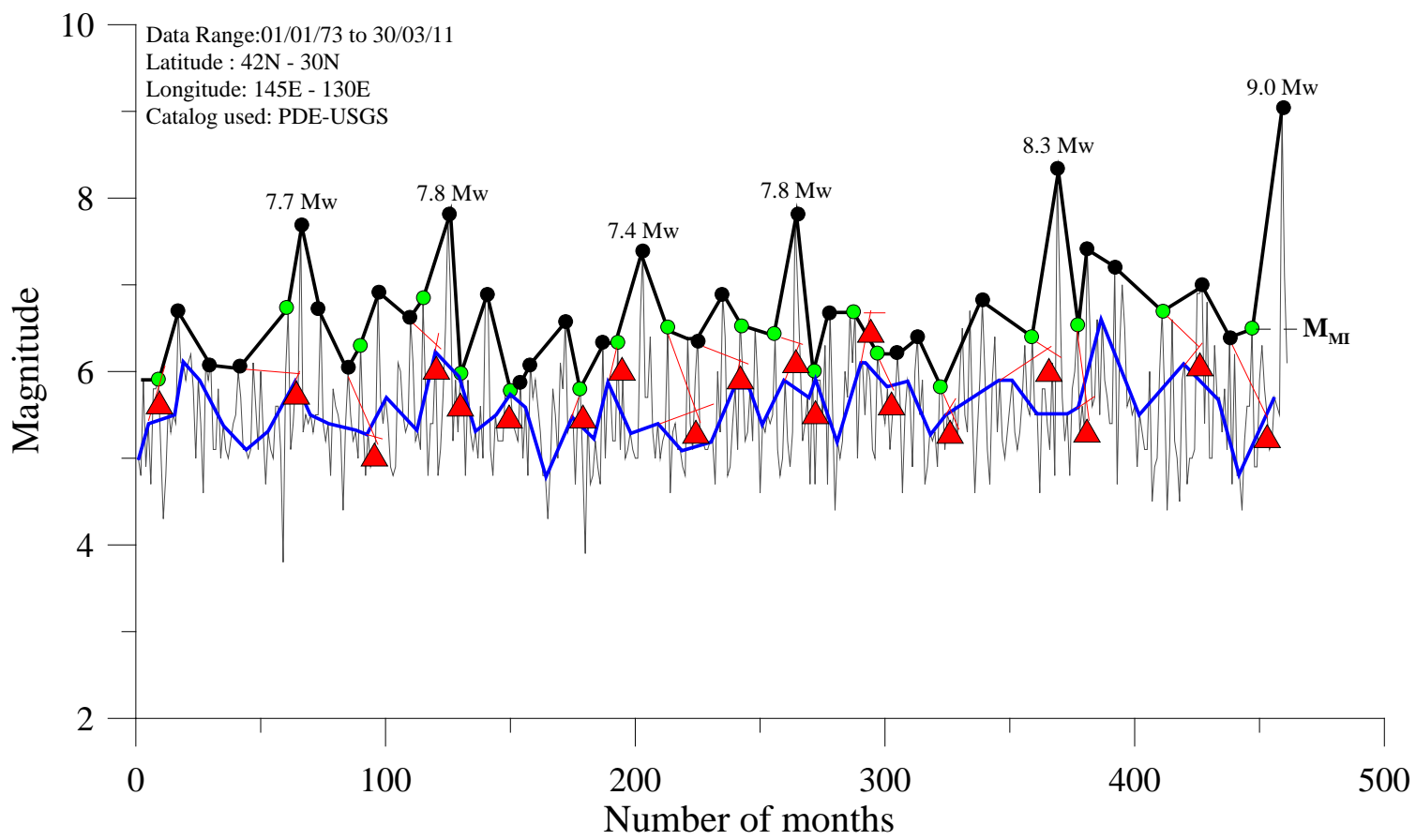

Figure 13. Warning signs (red triangles generated by the oscillation band of the extreme points-microsequence DB-3SE. 


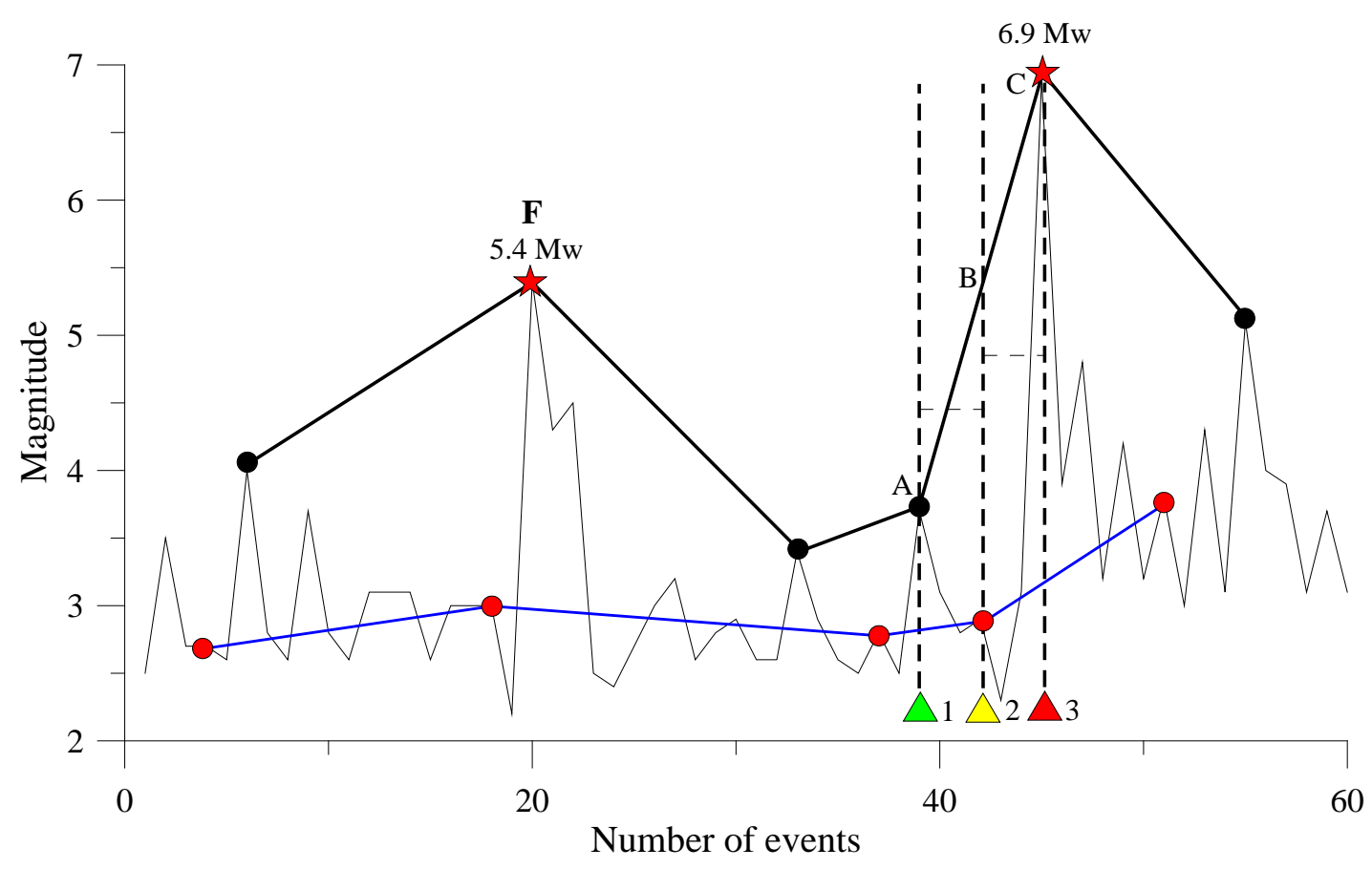

Figure 14. Warning signs before the earthquake in Loma Prieta, 1989. The red star indicates the mainshock, the letter "F" the foreshock.

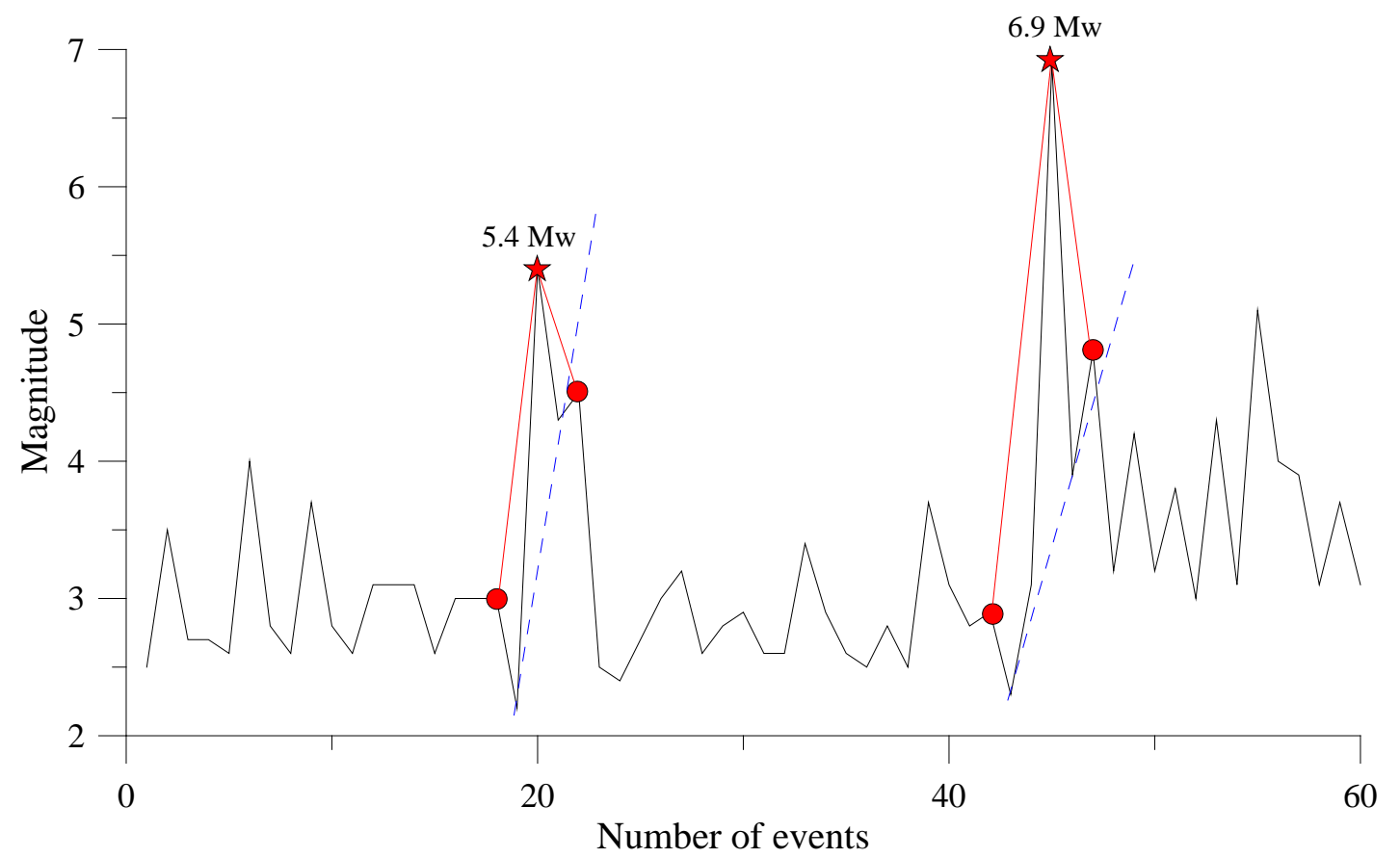

Figure 15. Microsequence TT-7S in energy release phase. The red symbols indicate the three maximum values in the microsequence.

coincides with the most energetic shocks (foreshock and mainshock), while the third maximum, which closes the energy release phase, is represented by an aftershock.

The transition from the energy release to the energy accumulation phase is also confirmed by the magnitude values decrease below the "transition line" (dashed blue line) [6]. 


\section{Conclusions}

A seismic sequence that occurs in a given area consists of microsequences and repetitive patterns formed by a grouping of seismic events with particular features. Their analysis allows studying the short-term evolution of a seismic sequence by identifying the warning signals that precede a strong earthquake.

In particular, the microsequence TT-7S allows us to locate the closure point of an energy release phase and identify a second order warning sign.

The microsequence DB-3SE, identifies the beginning of the energy release phase and explains how from low energy earthquakes moderate to high magnitude events can result, whose occurrence time can be known with sufficient advance.

The band allows us to understand when the seismic sequence is located within an energy release or accumulation phase; therefore if it reaches a critical stage where small shocks can trigger shocks of greater magnitude.

The aforementioned method, based on the microsequence analysis and on repetitive patterns can be easily applied to the study concerning earthquakes forecasts both at regional and global level. The method is based on a simple graphical method that can be used through the data of different seismicity catalogues.

\section{References}

[1] Wakita, H., Nakamura, Y. and Sano, Y. (2013) Case 3: Short-Term and Intermediate-Term Geochemical Precursors. In: Wyss, M., Ed., Evaluation of Proposed Earthquake Precursors, American Geophysical Union, 15-20. http://onlinelibrary.wiley.com/doi/10.1029/SP032p0015/summary

[2] Kanamori, H. (1981) The Nature of Seismicity Patterns before Large Earthquakes. In: Simpson, D.W. and Richards, P.G., Eds., Earthquake Prediction, American Geophysical Union, Washington, 1-19.

http://onlinelibrary.wiley.com/doi/10.1029/ME004p0001/summary http://dx.doi.org/10.1029/me004p0001

[3] Mantovani, E., Babbucci, D., Tamburelli, C. and Viti, M. (2009) A Review on the Driving Mechanism of the Tyrrhenian-Apennines System: Implications for the Present Seismotectonic Setting in the Central-Northern Apennines. Tectonophysics, 476, 22-40. http://dx.doi.org/10.1016/j.tecto.2008.10.032

[4] Riga, G. and Balocchi, P. (2016) Short-Term Earthquake Forecast with the Seismic Sequence Hierarchization Method. Open Journal of Earthquake Research, 5, 79-96. http://www.scirp.org/Journal/PaperInformation.aspx?PaperID=66332 http://dx.doi.org/10.4236/ojer.2016.52006

[5] Helmstetter, A., Kagan, Y.Y. and Jackson, D.D. (2005) Importance of Small Earthquakes for Stress Transfers and Earthquake Triggering. Journal of Geophysical Research: Solid Earth. http://onlinelibrary.wiley.com/doi/10.1029/2004JB003286/full

[6] Riga, G. and Balocchi, P. (2016) Seismic Sequence Structure and Earthquakes Triggering Patterns. Open Journal of Earthquake Research, 5, 20-34. http://www.scirp.org/Journal/PaperInformation.aspx?PaperID=63538 http://dx.doi.org/10.4236/ojer.2016.51003

[7] Bulkowski, T. N. (2002) Trading Classic Chart Patterns. John Wiley \& Sons, Inc. http://fx-arabia.com/vb/uploaded/178_01275676445.pdf

\section{Submit or recommend next manuscript to SCIRP and we will provide best service for you:}

Accepting pre-submission inquiries through Email, Facebook, Linkedin, Twitter, etc

A wide selection of journals (inclusive of 9 subjects, more than 200 journals)

Providing a 24-hour high-quality service

User-friendly online submission system

Fair and swift peer-review system

Efficient typesetting and proofreading procedure

Display of the result of downloads and visits, as well as the number of cited articles

Maximum dissemination of your research work

Submit your manuscript at: http://papersubmission.scirp.org/ 